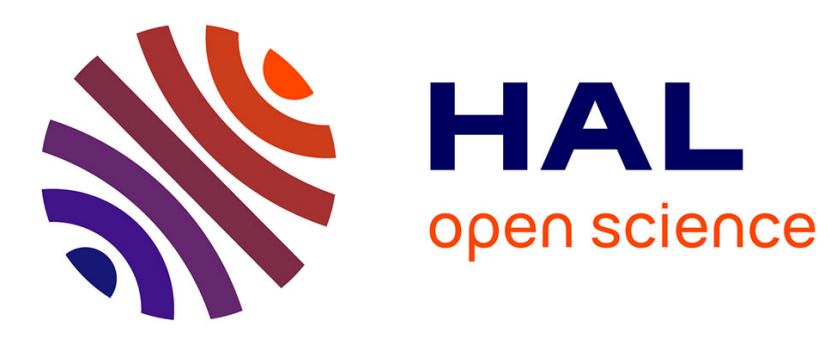

\title{
Simulation of Cellular Packing in Non-Proliferative Epithelia
}

Yoann Chélin, Karim Azzag, Patrick Cañadas, Julien Averseng, Stephen Baghdiguian, Bernard Maurin

\section{- To cite this version:}

Yoann Chélin, Karim Azzag, Patrick Cañadas, Julien Averseng, Stephen Baghdiguian, et al.. Simulation of Cellular Packing in Non-Proliferative Epithelia. Journal of Biomechanics, 2013, 46 (6), pp.1075-1080. 10.1016/j.jbiomech.2013.01.015i . hal-00799386

\section{HAL Id: hal-00799386 https://hal.science/hal-00799386}

Submitted on 12 Mar 2013

HAL is a multi-disciplinary open access archive for the deposit and dissemination of scientific research documents, whether they are published or not. The documents may come from teaching and research institutions in France or abroad, or from public or private research centers.
L'archive ouverte pluridisciplinaire HAL, est destinée au dépôt et à la diffusion de documents scientifiques de niveau recherche, publiés ou non, émanant des établissements d'enseignement et de recherche français ou étrangers, des laboratoires publics ou privés. 
A paper submitted to the Journal of Biomechanics

Title: Simulation of Cellular Packing in Non-Proliferative Epithelia

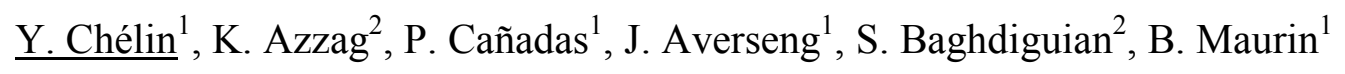

1. Université Montpellier 2, Laboratoire de Mécanique et Génie Civil, CNRS, Place Eugène Bataillon, 34095 Montpellier Cedex 5, France

2. Université Montpellier 2, Institut des Sciences de l'Evolution, CNRS, Place Eugène Bataillon, 34095 Montpellier Cedex 5, France

Article Type: Full Length Article (max 3000 words)

Word count: 2,929 (from Introduction through Discussion / from the Introduction through the Acknowledgments, including any appendices)

\section{Abstract:}

The physical laws governing the morphogenesis of biological tissues remain largely misunderstood. In particular, the role of the mechanical interactions occurring in this process needs to be better understood and studied. Inner follicular cells surrounding the oocytes of Ciona intestinalis form an epithelial monolayer resulting from an accretion process (without mitosis or apoptosis). This epithelium is elementary and useful for morphogenesis studies: the cells exhibit polygon packing with a specific but non-systematically repeatable topology (i.e. the distribution of pentagons, hexagons and heptagons changes). To understand the role of mechanical forces in tissue formation, we propose an innovative "2D spherical" model based on the physics of divided media. This approach simulates the cellular mechanical behavior and epithelium structuration by allowing cells to adopt a large variety of shapes and to selforganize in response to mechanical interactions. The numerical parameters considered in the model are derived from experimental data in order to perform pertinent and realistic simulations. The results obtained are compared to biological observations using the same counting method to characterize epithelium topology. Numerical and experimental data appear close enough to validate the model. It is then used for exploratory studies dealing with "Tissue Development Speed" variation, which is not easily attainable by experimentation. We show that the formation speed of the tissue influences its topology and hence its packing organization. 


\section{1. Introduction}

2 The question of how biological tissues are formed is a challenge for biology, and the causes

3 of cell organization in the course of epithelium development are misunderstood (Martinand-

4 Mari et al., 2009; Gibson et al., 2006; Classen et al., 2005; Mammoto and Ingber, 2010;

5 Glazier, 1989). The eggs of Asicidians, marine invertebrate hermaphroditic organisms,

6 constitute a classical model in biology development studies, the most commonly studied

7 being Ciona intestinalis (CI).

8 As shown by Martinand-Mari and collaborators, the oocyte surface of CI is progressively 9 covered by testa cells (Fig. 1; see also Martinand-Mari et al., 2009). These cells secrete a 10 specific extracellular matrix (called "chorion") on top of which two layers of follicular cells 11 (FCs) are deposited: internal FCs (IFCs) and outer FCs (OFCs). As the oocyte develops up to its final size, the follicular cells grow proportionally. This accretion mechanism, which drives folliculogenesis, is therefore complemented by a cellular period of growth controlled by the 14 growth of the oocyte itself (Fig. 2-a). The process continues up to the completion of the

follicular structure, characterized by about 60 OFCs forming a tight, compact coverage made up of 60 polygons (Fig 2-b). In fact, IFC topology appears to be univocally linked to that of OFCs. Based on these developmental and topological considerations, only the IFCs are taken into account in present study as in previous work (Martinand-Mari et al., 2009). Interestingly, the development of this specific follicular epithelium occurs without any cell division or apoptosis (Mancuso, 1965).

Tissue patterns of this type are generally characterized by counting the number of polygon sides of each cell, also corresponding to the number of neighboring cells (Gibson et al., 2006; Classen et al., 2005). It has been largely observed that this topology is not systematically repeatable: the numbers of hexagons, pentagons or heptagons vary slightly between different 
CI eggs while hexagons are always the major components.

This kind of specific topology is frequently observed in biology, as in the "perfect" hexagonal organization of honeycomb cell (or alveolus) as shown in Fig. 2-c. Such configurations are also studied in other scientific fields: fullerene carbon molecules with hexagonal and pentagonal sides, soap foam, etc. The morphogenesis of natural patterns comes from physicochemical mechanisms, mostly based on minimizing the system's potential energy. For example, the honeycomb is based on a "constrained structuration" process corresponding to self-equilibrium between the internal forces acting within the alveolus walls and external forces due to growth of larvae. The mechanical interactions result in a uniform tension system, which constitutes an optimal shortest path network (hexagons): this type of organization minimizes the amount of wax required to build a given number of alveolus, also minimizing structural energy (Thompson, 1917). Furthermore, the relationship between the biological function and the architectural organization of the structure of living cells and tissues has already been described by using a geodesic form analogy based on tension/compression equilibrium (Ingber, 1998).

Moreover, the distribution of polygons on a spherical surface corresponds to a historical question. Indeed, in 1752, Euler demonstrated the impossibility of totally covering a sphere using only hexagons: 12 pentagons are required in this case. As an example, $\mathrm{C}_{60}$ fullerene is formed by 20 hexagons and 12 pentagons (like a soccer ball).

Finally, although the relationships between mechanical forces and morphogenesis mechanisms, including mechanotransduction processes and the structure-function relationships, have been largely studied from the cellular level (Oster et al., 1983; Ingber et 
2009) to the organ scale (Thompson, 1917; Wirtz and Dobbs, 2000; Grodzinsky et al., 2000; Hou et al., 2007; Fleury, 2012), this is not yet the case for non-proliferative epitheliums like CI follicular tissues.

Indeed, to our knowledge, only one physical study has been published in this field (Martinand-Mari et al., 2009). The authors developed a model based on an analogy with a set of identical electrically charged "curved disks", freely moving on a spherical surface. The resulting balanced configuration corresponds to a specific distribution of 48 hexagons and 12 pentagons (consistent with Euler's theorem), whose edges connect the disk centers. It is also systematically repeatable and based on tetrahedral symmetry. Even if comparisons with observed CI epitheliums show that their cells tend to organize themselves in this way, the pattern uniqueness of this analogy is not adapted to the non-reproducibility of biological epitheliums.

Accordingly, our main objective is to propose a new morphogenesis model of the IFC epithelium, able to bring out the role of the mechanical stresses in the accretion process and to generate patterns like those experimentally observed. Furthermore, this model is intended to enable predictive studies to test the incidence of factors governing epithelium morphogenesis.

To do so, we use modeling with the least possible constraints to produce a high level of selforganization in the evolution of the system. Indeed, it is important to obtain numerical results with low dependency on deterministic rules or initial hypothesis imposed by the model. It is noteworthy that, numerical simulations based on the physics of divided media have already shown their ability to represent a large variety of dynamical reorganizations of the cytoplasm of adherent cells (Milan et al., 2007; Maurin et al., 2008; VanLiedekerke et al., 2010). 
77 We thus developed a new "granular" model applied to CI epithelial morphogenesis, in which individual cells correspond to the IFCs. In the simulations, cells evolve on a spherical surface in accordance with their physical properties and mechanical interactions. They consequently self-organize, leading to specific final tissue patterns. The model we developed is driven by biological data to obtain numerical morphogenesis consistent with experimental observations (final number of cells and sizes of cells and oocytes).

\section{Methods}

In the model, each cell is composed of two types of "grain" moving on a sphere surface ("2D spherical" representation). The first type represents the inner cytoplasm without an accurate description of the cytoskeleton structure. Even if its filaments play a major stress-bearing role, our biological observations on CI oocytes show that actin filaments (main cytoskeleton tension-bearing filaments) are essentially organized as peripheral structures and that no stress fiber is revealed within the cytoplasm (Fig. 2). The resulting assumption made here considers that a global balance is obtained between tensile peripheral forces and compressive forces inside the cell. Accordingly, as a first approximation, our model assumes that pressure in the cell produced by repulsive internal interactions is equilibrated by peripheral tension born by membrane elements. Thus, the second type of grain, linked by these cable elements, represents the membrane associated with the actin cortex. The forces exerted by these cables are combined with repulsive action between grains and both govern the mechanics of the media. Moreover, interactions between the membrane cables and the internal grains cause them to remain inside the cell. All the interactions are managed by the molecular dynamic method (Allen and Tildesley, 1987) extended to the grain/cable contacts, including normal viscous interactions but not adhesion or friction. Indeed, we decided to consider only unidirectional contact interactions, i.e. normal forces without any tangential (or friction) 
101 forces, as can be done in granular mechanics (F. Radjaï and F. Dubois, 2011). Thus, viscosity

102 is only linked to normal velocity here, as in rheology when considering the Kelvin-Voigt 103 model. The numerical processing chosen here to describe the contacts allows grain

104 interpenetration, as is visible in Fig. 3 where some grains look like polygons. By considering

105 (S) as the dynamical state of the system at time $t$ (position and velocity of all the grains), we

106 use a Runge-Kutta RK4 explicit time integration scheme for the function $f(S, t)$, which

107 represents the temporal evolution of the system governed by the mechanical equations of 108 solid dynamics:

109

$$
f(X, t)=\frac{d X(t)}{d t}=\left[\begin{array}{lll}
\dot{x}_{1}(t) & \ldots & \dot{x}_{g}(t) \\
\ddot{x}_{1}(t) & \ldots & \ddot{x}_{g}(t)
\end{array}\right]=\left[\begin{array}{ccc}
\dot{x}_{1}(t) & \ldots & \dot{x}_{g}(t) \\
F_{1} / m_{1} & \ldots & F_{g} / m_{g}
\end{array}\right]
$$

111 where $x_{g}(t), \ddot{X}_{g}(t)$ and $\mathbb{W}_{g}(t)$ are respectively the position, velocity and acceleration of an

112 individual grain $g$, with $F_{\mathrm{g}}$ equal to the sum of the forces applied to it and $m_{\mathrm{g}}$ its mass.

114 Each cell grows consecutively as new grains are generated inside it successively by considering five different diameters coming up periodically (Fig. 3-a). This sequential process gives a heterogeneous cytoplasm to better allow dynamical reorganization (Table 1). Cell

117 growth occurs until a maximum size of the cell is reached, corresponding to a maximum 118 number of internal grains.

120 In this process, membrane tension $(T)$ should increase with cell growth. However, previous

121 authors have already reported that cortical actin tension changes slightly when epithelial cells 122 are treated by adding cytochalasin D (Laurent et al., 2003). So the membrane tension is 
123 restricted here between two values $\left(T_{\min }\right.$ and $T_{\max }$, determined empirically to reach system equilibrium) by controlling the free length $\left(L_{\mathrm{f}}\right)$ of the cable as follows: $L_{\mathrm{f}}=\left(E_{m} A_{m} L\right) /\left(E_{m} A_{m}+T_{\max }\right)$ in order to decrease $T$;

- If a cable element with length $L$ has a tension $T<T_{\min }$, then $L_{\mathrm{f}}$ decreases as

$$
L_{\mathrm{f}}=\left(E_{m} A_{m} L\right) /\left(E_{m} A_{m}+T_{\min }\right) \text { in order to increase } T \text {; }
$$

129 where $E_{\mathrm{m}}$ and $A_{\mathrm{m}}$, both considered constant, are respectively the tensile elasticity modulus

130 (see Table 1) and the cross-section area of the membrane cable. Note that tensions $T_{\min }$ and

$131 T_{\max }$ correspond to minimum and maximum values of cable strain: $\varepsilon_{\min }=T_{\min } /\left(E_{m} A_{m}\right)$ and

$132 \varepsilon_{\max }=T_{\max } /\left(E_{m} A_{m}\right)$. Consequently, adjusting the free length $L_{\mathrm{f}}$ of cable element in order to

133 control its tension, maintains its strain in the predetermined range $\left[\varepsilon_{\min } ; \varepsilon_{\max }\right]$.

134 In addition, if a cable length is too great $\left(L>L_{\max }\right)$, it is divided into two cable elements.

135 Conversely, if the lengths of two consecutive elements are too small $\left(L<L_{\min }\right)$, they are 136 merged.

Adding new grains and lengthening membrane-cables physically correspond to new incoming material. Hence, this model refers to an open-mass system. This assumption is biologically

140 corroborated by the tissue growth produced by nutrients in the oocyte environment, as 141 experimentally observed.

142 As a result, a numerical cell with adaptive membrane tension and a polydisperse cytoplasm 143 can be deformed into a wide variety of shapes (shape "plasticity"). It can also move freely on 144 the oocyte spherical surface and "push" other cells via membrane contacts (Fig. 3). Thus, 145 cells can self-organize leading to unique tissue topologies. Statistical analysis was performed 146 at the end of the simulations using the same method as in experimental studies. 
148 At the beginning of a simulation, 10 small cells are randomly positioned on the oocyte (note

149 that the image (a) in figure 4 does not represent this initial configuration but a stage shortly

150 after). New cells arrive regularly and are randomly disposed on the sphere, provided there is

151 enough free space, while the oocyte diameter increases. In the same time, all the cells grow

152 (Fig. 4).

153

154 The dynamic evolution of the model is thus driven by different speeds: accretion speed (Vac) 155 corresponding to the arrival of new cells, cell growing speed $(V c g)$ and oocyte growing speed

156 (Vog). These three speeds were empirically adjusted from biological observations by 157 considering both the number and size of the cells at different times: cells are small at the 158 beginning and become bigger until they reach their maximum size at the maturation stage of 159 the oocyte. The model hence proposes an equivalent numerical evolution, leading to a 160 confluent monolayer of about 60 cells, coupled with increasing egg diameter (from 90 to 160 $161 \mu \mathrm{m}$ in the case of CI). Taken together, these three speeds (Vac, Vcg and $V o g$ ) define a "Tissue 162 Development Speed".

163 Simulation is stopped when IFCs cover the whole surface. The number of cells in contact

164 (membrane/membrane) is then counted for each cell to determine the topology.

165 Tissue developments are not identical since simulations are doubly unpredictable. Cells are 166 randomly positioned on the oocyte surface twice (for native cells and new incoming ones) and 167 their growth combined with their interactions, influence their shapes and the overall form of 168 the resulting tissue. The dynamical processes and the unpredictability of cell positions drive 169 the packing of the virtual epithelium and, in consequence, tissue morphogenesis.

172 Variation of only one of the three speeds driving tissue formation, while the two others 
173 remain unchanged, leads to unsuitable tissue: at the final stage, it is composed of too few cells

174 (the tissue is not confluent) or too many cells (the calculations never reach a stable

175 equilibrium). Simulations were therefore performed by varying "Tissue Development Speed"

176 (TDS). A "reference" TDS was determined to obtain the closest results to experimental

177 observations: values of $V a c, V c g$ and $V o g$ were adjusted in the "trial and error" process

178 explained previously. We also considered a "slow" TDS (where speeds of the "reference"

179 TDS are divided by two) and a "fast" TDS ("reference" values are multiplied by two) as

180 shown in Table 2. Ten simulations were performed for each TDS.

182 The average number of computed IFCs is respectively $58.0(+/-1.9), 58.8(+/-1.4)$ and 60.7

183 (+/- 1.0) for "slow", "reference" and "fast" TDS. Experimentally, an average number of 58.7

184 (+/- 1.8) was measured (Martinand-Mari et al., 2009), which is consistent with simulations

185 (Fig. 5). Interestingly, raising the TDS results in a slight increase in the final number of IFCs

186 on the oocyte. This may be explained by the fact that, when the TDS is higher, the IFCs

187 already positioned on the surface have less time to grow before new cells arrive by accretion.

188 Geometrically speaking, there are more available empty spaces between the growing IFCs,

189 sometimes allowing more new IFCs to arrive on the sphere.

191 In addition, all the numerical tissues were composed of confluent IFCs with pentagonal,

192 hexagonal and heptagonal shapes, exceptionally tetragonal or octagonal (Figs. 4 and 5). The

193 numbers of polygons were counted as previously described for each simulation and the

194 average values were calculated for each TDS. Fig. 5 presents these average distributions

195 showing good agreement with experimental observations made on ten oocytes by confocal

196 microscopy (Martinand-Mari et al., 2009). Furthermore, tissue computed with the slow TDS

197 had more hexagonal and fewer non-hexagonal cells, in comparison with the results produced 
by fast TDS.

199 Quantitatively, the average experimental topology was roughly 17 pentagons (29.7\%), 37 200 hexagons $(63.2 \%)$ and 4 heptagons $(7.1 \%)$. It is interesting to note that this organization 201 differs from Euler's distribution for 60 polygons, i.e. 48 hexagons (80\%) and 12 pentagons $202(20 \%)$ that could be related to the results obtained in studies like (Martinand-Mari et al., 203 2009). The morphogenetic process of accretion leads, in the case of IFCs of Ciona 204 intestinalis, to another configuration in which a key point may be "optimum" physical 205 interaction energy. In fact, Martinand-Mari and coworkers used always 60 identical disks and do not consider the role of mechanical forces between IFCs in their growing and spatial rearrangement which always converge to the same distribution of neighbors (12 disks with 5 208 neighbors and 48 with 6 neighbors; personal communication of Martinand-Mari co-author S. 209 Baghdiguian). By contrast, our model takes into account individual cell behaviors and is driven by the mechanical interactions between cells, leading to tissues formed by a number of 211 cells varying from $58.0(+/-1.9)$ to $60.7(+/-1.0)$ and a fluctuating number of cell neighbors.

212 Accordingly, mechanical forces can govern the specific morphogenesis of IFC epithelium, its

213 "final" state corresponding to a balanced system.

214 Numerical simulations with slow TDS also tended to be closer to Euler's packing (the 215 contrary being seen for fast TDS). Indeed, a decrease in TDS may give the cells more time to 216 self-organize, thus getting closer to this packing.

217 Moreover, topology varied slightly in the simulations for the same TDS. This shows that the 218 model gives non-systematic results. However, we noted that the numerical standard 219 deviations were low (maximum 6.6\%) and close to experimental values (maximum 9.4\%). 220 This indicates that morphogenesis may be a fairly stable mechanism. Deviations also seem to 221 increase slightly with TDS: a slow value gives better repeatability than a fast one (Table 3 ). 222 This is consistent with the above comments on the relationships between IFC organization 
and overall time evolution.

To sum up, the present model is able to reproduce tissue organization as biologically observed and to predict the possible influence of "Tissue Development Speed" on a non-proliferative epithelium. The implemented numerical growth of both cells and oocyte drives the dynamical evolution of the tissue, providing a means of analyzing the mechanical forces inside and between the cells during the morphogenesis process. However, in spite of good agreement

231 with experimental results, particularly concerning polygon distributions, we observe that 232 numerical standard deviations were always lower than experimental ones. This suggests that 233 other biological features (nutriment concentration, biochemical cascades, signalling 234 transduction, etc.) and structures (adhesion protein complexes, cytoskeleton, testa cells and 235 OFCs, etc.) may influence the development of IFCs. Accordingly, our model could be 236 improved by including some of these aspects.

237 A major drawback of this approach is that cellular modeling is too basic, being limited to 238 intracellular pressure, produced by repulsion between the inner grains, and surrounded by a 239 peripheral tension carried by the membrane-cables. However, previous studies have shown 240 that granular cell modeling may also provide a means of considering a dynamical 241 cytoskeleton (Milan et al., 2007; Maurin et al., 2008). Thus, one possible development could 242 be to implement such a dynamical "granular cytoskeleton", thus producing a macro-micro 243 description of tissue morphogenesis (Sandersius and Newman, 2008) for 244 mechanotransduction analyses. Similarly, the model could be enhanced by including 245 intercellular links such as cadherins. In addition, other morphogenesis simulations could be 246 conceived using different geometries (e.g., a planar substrate), different boundary conditions 247 (e.g., a circular or a rectangular box) or other mechanisms that govern morphogenetic 
processes including proliferation or apoptosis (Dubertret and Rivier, 2000).

249 Overall, this promising approach, based on an original cell-tissue modeling, allows new useful architectural descriptions and mechanical analysis of cell and tissue evolution in 251 epithelial morphogenesis.

\section{References}

254 Allen, M., Tildesley, D., 1987. Computer simulation of liquids. Oxford university Press, 255 Oxford.

Classen, A.-K., Anderson, K.I., Marois, E., Eaton, S., 2005. Hexagonal packing of Drosophila wing epithelial cells by the planar cell polarity pathway. Developmental Cell 9, 805-817.

Thompson, D.W., 1917. On growth and form. Cambridge University Press, Cambridge.

Dubertret, B., Rivier, N., 2000. Geometrical models of the renewal of the epidermis. Comptes Rendus de l'Académie des Sciences III 323, 49-56.

Fleury, V., 2012. Clarifying tetrapod embryogenesis by a dorso-ventral analysis of the tissue 266 flows during early stages of chicken development. Biosystems 109, 460-474.

Gibson, M.C., Patel, A.B., Nagpal, R., Perrimon, N., 2006. The emergence of geometric order in proliferating metazoan epithelia. Nature 442, 1038-1041.

Glazier, J., 1989. Dynamics of cellular patterns. PhD. thesis, University of Chicago, Chicago. 
273 Grodzinsky, A.J., Levenston, M.E., Jin, M., Frank, E.H., 2000. Cartilage tissue remodeling in 274 response to mechanical forces. Annual Review of Biomedical Engineering 2, 691-713.

276 Hou, B., Fukai, N., Olsen, B.R., 2007. Mechanical force-induced midpalatal suture 277 remodeling in mice. Bone 40, 1483-1493.

Ingber, D.E., Dike, L., Hansen, L., Karp, S., Liley, H., Maniotis, A., McNamee, H., Mooney, D., Plopper, G., Sims, J., 1994. Cellular tensegrity: exploring how mechanical changes in the cytoskeleton regulate cell growth, migration, and tissue pattern during morphogenesis. International Review of Cytology 150, 173-224.

Ingber, DE., 1998. The Architecture of Life. Scientific American 278, 48-57.

Lecuit, T., Lenne, P.-F., 2007. Cell surface mechanics and the control of cell shape, tissue patterns and morphogenesis. Nature Reviews: Molecular Cell Biology 8, 633-644.

Mammoto, T., Ingber, D.E., 2010. Mechanical control of tissue and organ development. Development 137, 1407-1420.

Mancuso, V., 1965. An electron microscope study of the test cells and follicle cells of Ciona 293 intestinalis during oogenesis. Acta Embryologiae et Morphologiae Experimentalis 8, 239-266.

295 Martinand-Mari, C., Maury, B., Rousset, F., Sahuquet, A., Mennessier, G., Rochal, S., 296 Lorman, V., Mangeat, P., Baghdiguian, S., 2009. Topological control of life and death in non297 proliferative epithelia. PLoS One 4, e4202. 
299 Maurin, B., Cañadas, P., Baudriller, H., Montcourrier, P., Bettache, N., 2008. Mechanical 300 model of cytoskeleton structuration during cell adhesion and spreading. Journal of 301 Biomechanics 41, 2036-2041.

303 Milan, J.L., Wendling-Mansuy, S., Jean, M., Chabrand, P., 2007. Divided medium-based 304 model for analyzing the dynamic reorganization of the cytoskeleton during cell deformation. 305 Biomechanics and Modeling in Mechanobiology 6, 373-390.

306

307 Nienhaus, U., Aegerter-Wilmsen, T., Aegerter, C.M., 2009. Determination of mechanical 308 stress distribution in Drosophila wing discs using photoelasticity. Mechanisms of 309 Development 126, 942-949.

311 Oster, G., Murray, J., Harris, A., 1983. Mechanical aspects of mesenchymal morphogenesis. 312 Journal of Embryology and Experimental Morphology December; 78, 83-125.

314 Radjaï, F., Dubois, F., 2011. Discrete-element Modeling of Granular Materials. ISTE 315 Ltd./Wiley, London.

316

317 Sandersius, S.A. Newman, T.J., 2008. Modeling cell rheology with the Subcellular Element 318 Model. Physical Biology 5(1), 015002.

320 Van Liedekerke, P., Tijskens, E., Ramon, H., Ghysels, P., Samaey, G., Roose, D., 2010. 321 Particle-based model to simulate the micromechanics of biological cells. Physical Review E 322 $81,061906$. 
324 Wirtz, H.R. Dobbs, L.G., 2000. The effects of mechanical forces on lung functions.

325 Respiration Physiology 119, 1-17.

326

327 


\section{Conflict of interest statement}

330 The authors disclose any financial and personal relationships with other people or

331 organizations that could inappropriately influence their work.

332 This includes no conflict of interest including employment, consultancies, stock ownership,

333 honoraria, paid expert testimony, patent applications/registrations, and grants or other 334 funding.

336 Non-publication statement

337 The authors state that this material has not been and will not be submitted for publication 338 elsewhere.

339

340 
Fig. 1. (a) Representation of a mature Ciona intestinalis eggs where inset corresponds to the field shown in (b). (b) Cross-sectional sketch showing the oocyte (O) surrounded by the testa cells (T), the extra cellular matrix called chorion (C) and both the inner (IFC) and outer (OFC) follicular cells. Extracted from Martinand-Mari et al., 2009.

Fig. 2. ( $a$ and $b$ ) Basal face of IFC covering a CI oocyte (labeled by FITC Phaloidin, revealing actin filaments): (a) non-mature egg during the accretion process and (b) mature egg with IFC polygonal organization. Note that actin filaments appear essentially organized as peripheral structures (actin cortex) and that no stress fiber is revealed. (c) Hexagonal topology of honeycomb (Thompson, 1917).

Fig. 3: (a) Cell growth process with increasing number of cytoplasmic-grains and membrane lengthening. (b) Cell-cell interactions: membrane-cables in contact define the organization between cells. Grain shapes often appear polygonal due to contact processing, which allows interpenetration.

Fig. 4: Steps (from (a) to (d)) of evolution of the follicular system surrounding the oocyte: shortly after the initial configuration (a); new cells arrive and the cells and oocyte grow simultaneously (b) and (c); development continues until the egg is totally covered (d). The image (e) corresponds to (d) but with the tissue membranes only to better reveal the topology). The number of neighbors of each cell is indicated at its center ((d) and (e)). A corresponding movie is available at http://www.lmgc.univ-montp2.fr/ canadas/Movie.

Fig. 5: Distribution of the average number of neighboring cells determined from experimental observations and simulations. Standard deviations increase slightly when TDS increases.

Table 1: Numerical values considered in simulations. $E_{\mathrm{gg}}$ and $C_{\mathrm{gg}}$ are respectively the compressive stiffness and damping of the grain-grain contact. $E_{\mathrm{gc}}$ and $C_{\mathrm{gc}}$ are respectively the compressive stiffness and damping of the grain-cable contact

Table 2: Relative values of the three "Tissue Development Speeds".

Table 3: Average values (standard deviation in parenthesis) of experimental and numerical polygon distributions. 
Figure 1

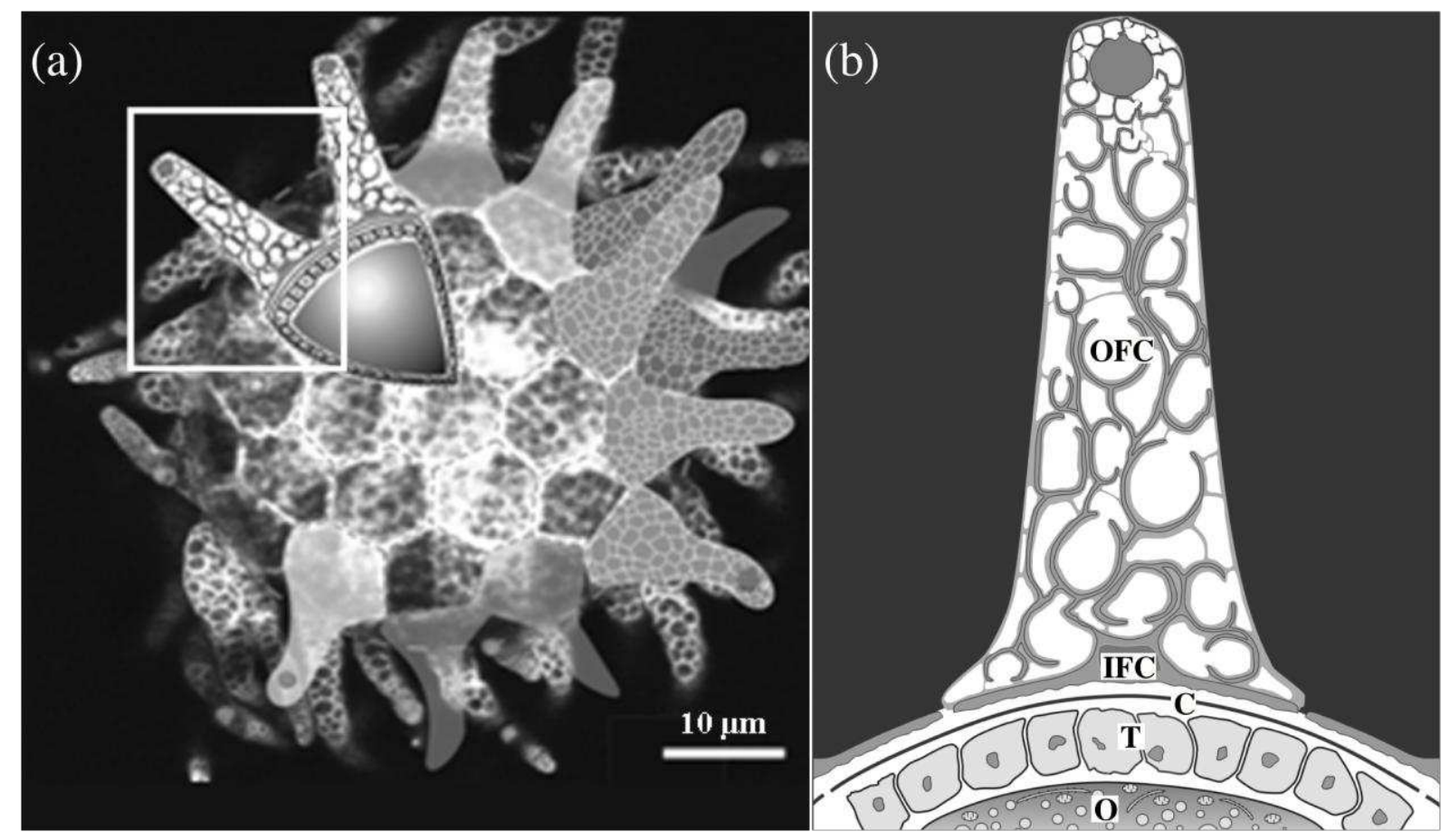


Figure 2

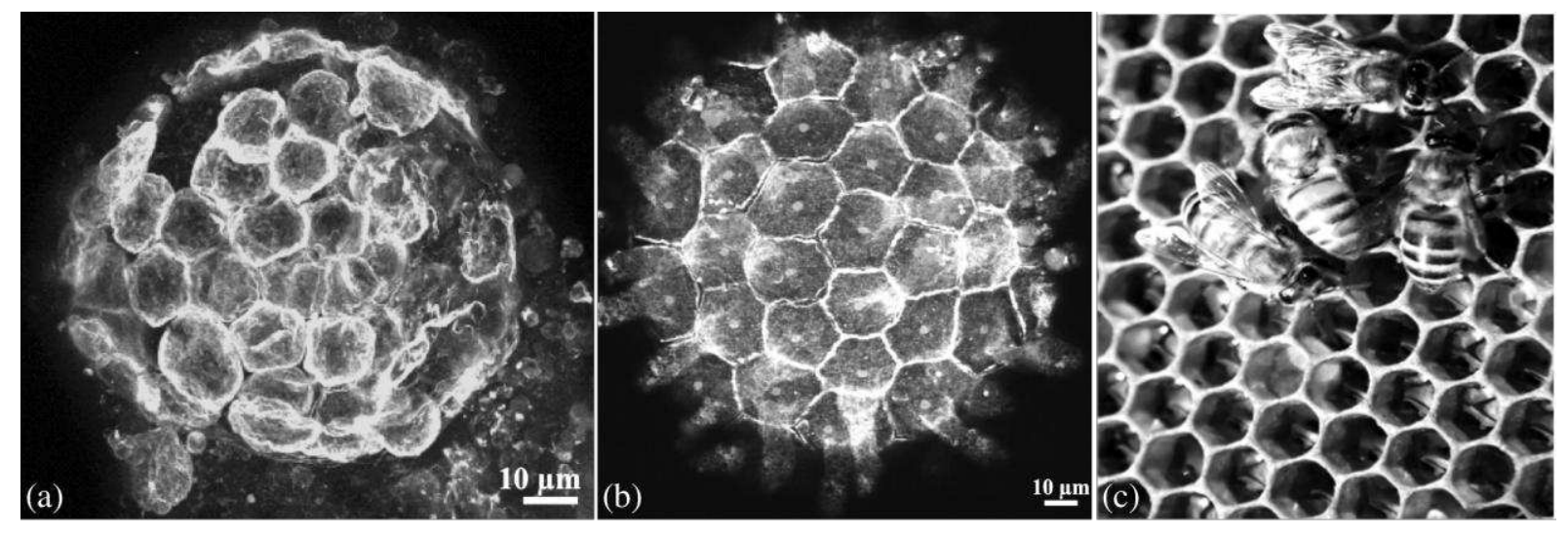


Figure 3

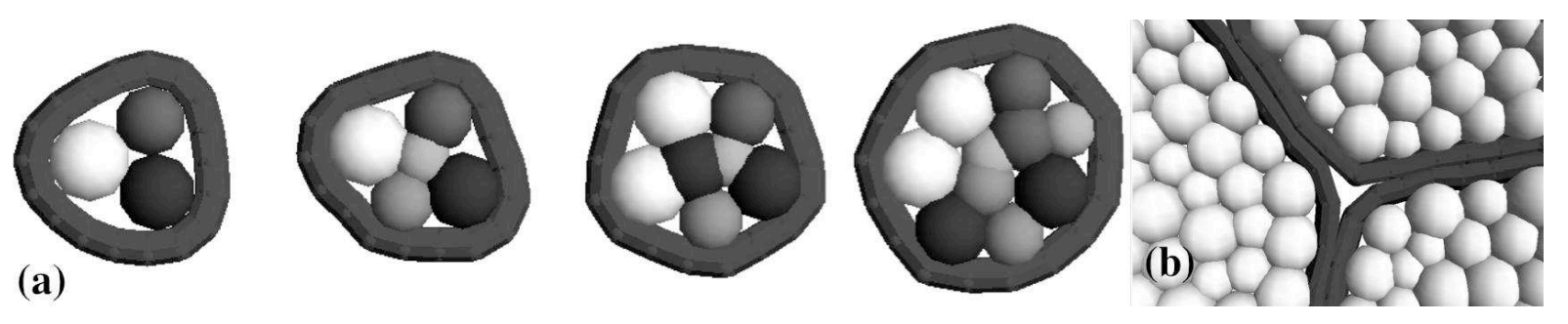


Figure 4

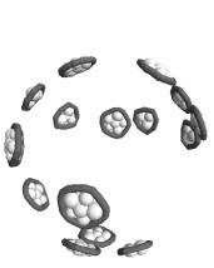

(a)

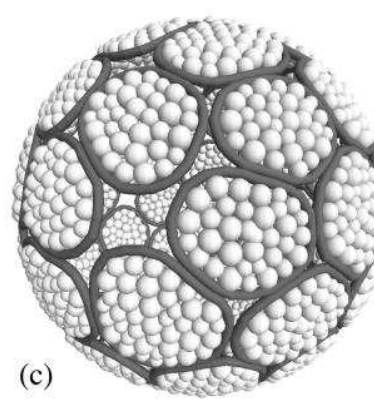

(b)

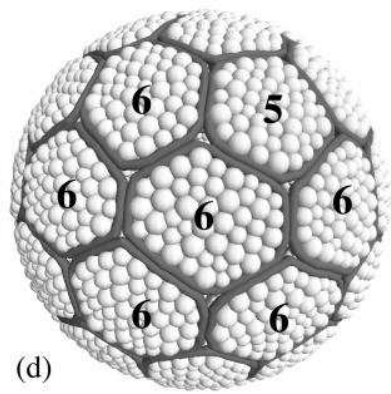

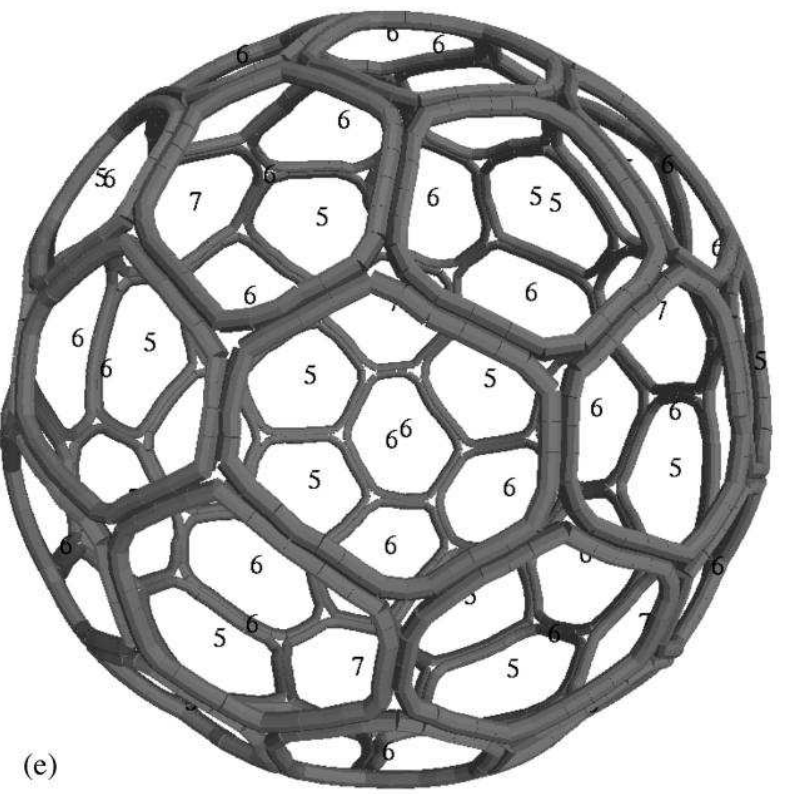


Figure 5

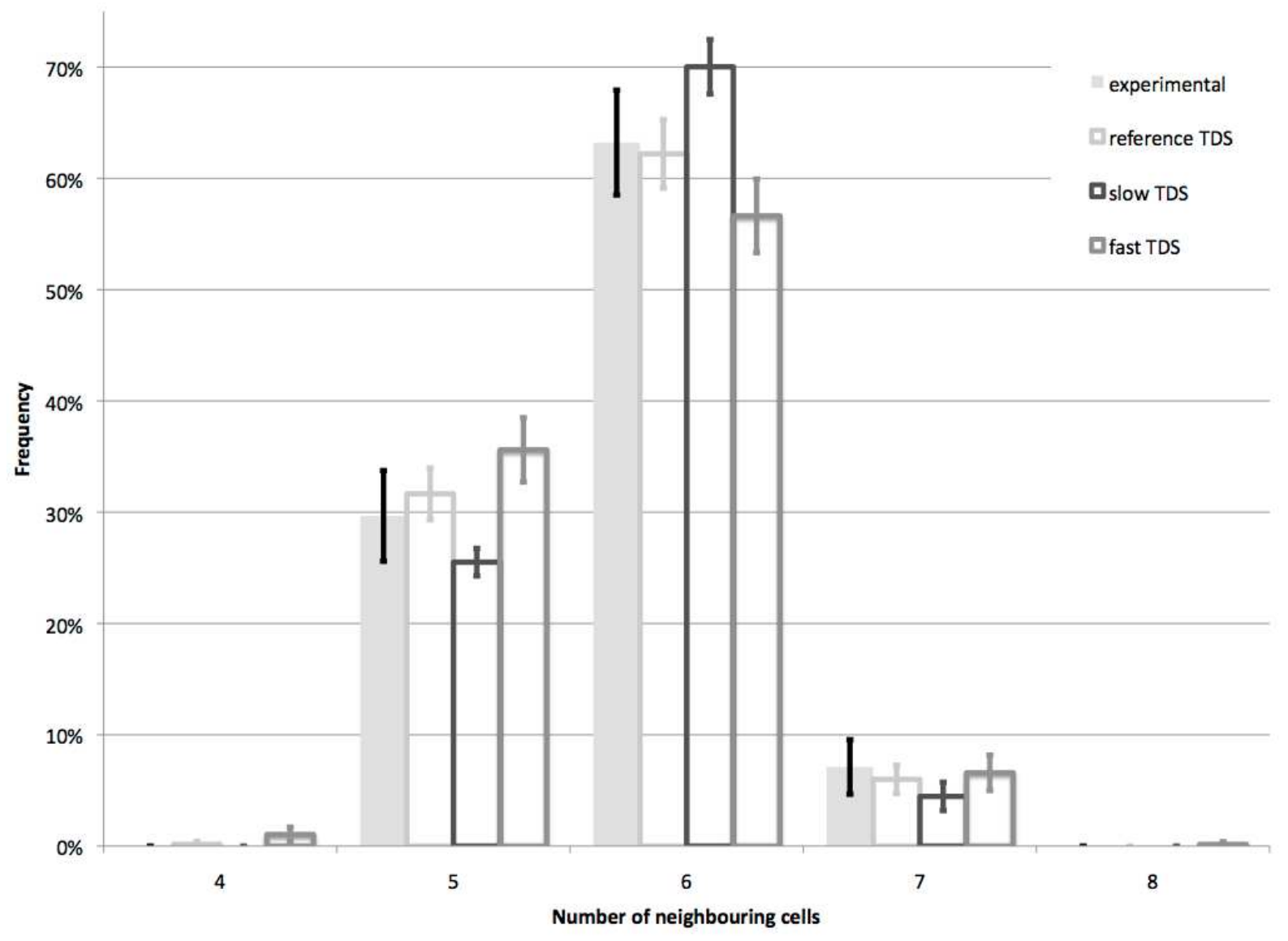




\section{Table 1}

\begin{tabular}{|c|c|}
\hline \multicolumn{2}{|l|}{ Geometry } \\
\hline - cytoplasmic-grain: size & diameter of grain $=5.0-5.6-6.0-6.6-7.0 \mu \mathrm{m}$ (sequential) \\
\hline number in a cell & - 3 grains at the beginning \\
\hline & - 45 grains at the end (for a mature cell) \\
\hline - membrane-cable: size & diameter $=3.0 \mu \mathrm{m}$ \\
\hline length & $L_{\min }=2.0 \mu \mathrm{m} ; L_{\max }=5.5 \mu \mathrm{m}$ \\
\hline \multicolumn{2}{|l|}{ Mechanics } \\
\hline - grain/grain contact: compressive stiffness and damping & $E_{\mathrm{gg}}=20 \mathrm{~N} / \mathrm{m} ; C_{\mathrm{gg}}=5 \mathrm{~N} /\left(\mathrm{m} \cdot \mathrm{s}^{-1}\right)$ \\
\hline - grain/cable contact: compressive stiffness and damping & $E_{\mathrm{gc}}=13 \mathrm{~N} / \mathrm{m} ; C_{\mathrm{gc}}=5 \mathrm{~N} /\left(\mathrm{m} \cdot \mathrm{s}^{-1}\right)$ \\
\hline - membrane-cable: tensile stiffness modulus & $E_{\mathrm{m}}=5.10^{-3} \mathrm{~N} / \mathrm{m}^{2}$ \\
\hline tension range & $T_{\min }=0.5 \mathrm{~N} ; T_{\max }=0.6 \mathrm{~N}$ \\
\hline \multicolumn{2}{|l|}{ Biology } \\
\hline \multirow[t]{2}{*}{ - cell growth } & grains added one by one \\
\hline & (their diameter depend on the number of existing grains) \\
\hline \multirow[t]{2}{*}{ - tissue growth } & new cells arrive one by one \\
\hline & (if there is enough space after testing 100 random positions) \\
\hline \multicolumn{2}{|l|}{ Numerical } \\
\hline - time step: used in $4^{\text {th }}$ order Runge-Kutta scheme & $0.005 \mathrm{~s}$ (see eq.1) \\
\hline
\end{tabular}


Table 2

\begin{tabular}{r|ccc} 
& reference TDS & slow TDS & fast TDS \\
\hline accretion speed & $V a c$ & $V a c / 2$ & $V a c \times 2$ \\
cell growth speed & $V c g$ & $V c g / 2$ & $V c g \times 2$ \\
oocyte growth speed & $\operatorname{Vog}$ & $V o g / 2$ & $\operatorname{Vog} \times 2$
\end{tabular}




\section{Table 3}

\begin{tabular}{c|ccccc} 
& tetragon & pentagon & hexagon & heptagon & octagon \\
\hline experimental & $0.0 \%( \pm 0.0)$ & $29.7 \%( \pm 8.1)$ & $63.2 \%( \pm 9.4)$ & $7.1 \%( \pm 4.9)$ & $0.0 \%( \pm 0.0)$ \\
\hline reference TDS & $0.2 \%( \pm 0.5)$ & $31.7 \%( \pm 4.7)$ & $62.2 \%( \pm 6.1)$ & $6.0 \%( \pm 2.5)$ & $0.0 \%( \pm 0.0)$ \\
slow TDS & $0.0 \%( \pm 0.0)$ & $25.5 \%( \pm 2.5)$ & $70.0 \%( \pm 4.9)$ & $4.5 \%( \pm 2.5)$ & $0.0 \%( \pm 0.0)$ \\
fast TDS & $1.0 \%( \pm 1.3)$ & $35.6 \%( \pm 5.8)$ & $56.6 \%( \pm 6.6)$ & $6.6 \%( \pm 3.2)$ & $0.1 \%( \pm 0.5)$
\end{tabular}

